\title{
Guillermo Hurtado, Por qué no soy falibilista y otros ensayos filosófico, México: Los Libros de Homero 2009, 87 pp.
}

Recopilar ensayos en un trabajo editorial generalmente implica que los mismos compartan un eje temático. Por qué no soy falibilista y otros ensayos filosóficos de Guillermo Hurtado no es el caso. Aunque el título provoca enfrentar ensayos sobre «doxología», nos encontramos con una labor más compleja: enfrentarnos a los procesos vitales/intelectuales del autor: «[...] los ensayos aquí incluidos son, sobre todo, el producto de un diálogo hondo conmigo mismo. No fueron escritos siguiendo modas o buscando ecos. Son el testimonio de un recorrido intelectual $y$, en el fondo, de un proceso vital» (p. 11).

De esta manera el libro se puede dividir en dos momentos biográficos; cada uno conformado por tres ensayos. En el primer momento podemos notar tres constantes temáticas: el cambio, los modos y la identidad personal. Todo esto en un plano meramente ontológico. El segundo momento pertenece principalmente a la «doxología»: falibilismo y sentido común; usos lingüísticos de «duda», «creencia» $\mathrm{y}$ «sospecha»; 
y una lectura contemporánea y laica sobre las aportaciones agustinianas a la teoría general de la creencia.

Decir que hay dos momentos biográficos en el texto no sugiere, en el caso particular de Guillermo Hurtado, que uno le sea inconexo al otro. $\mathrm{O}$ bien la relación entre ambos muestra un orden progresivo en los intereses intelectuales del autor, o el cuidado de la edición cuenta con una constancia placentera en cuanto al acomodo de los ensayos. Sea la opción que sea, lo seguro es que el texto de Hurtado deja al lector con un buen sabor de boca.

El acomodo de los textos parte de manera venturosa con «¿Qué es un cambio?» donde se estudia a fondo las dos concepciones aristotélicas de dicho concepto (Metabolé y Kinesis); se muestra que una es más fundamental que la otra, y se propone, alejándose de manera elegante del aristotelismo estricto, una concepción de cambio basada en una forma lógicotemporal. En su parte final, se defiende que una «descripción completa de un mundo dinámico como el nuestro requiere de, por lo menos, tres categorías ontológicas: estados, cambios y procesos» (p. 21). Los cambios, de esta manera, son un tipo de conjunciones de estados; mientras que los procesos, son un tipo de conjunciones de cambios. Dicha postura resulta provocativa en contraste con algunas lecturas davidsonianas, donde se sostiene que en el mundo no hay hechos (en este caso estados), sino sólo sucesos (cambios). Esto, para Hurtado, resulta insostenible ya que sin estados, no hay cambios. Repecto a Aristóteles, Hurtado señala que mientras el estagirita decía que el tiempo es la forma del cambio, el tiempo debe concebirse como el que da forma al cambio por encima de una forma previa, que es la conjuntiva (ver p. 19).

El segundo ensayo titulado «Entes y modos en las Disputationes Metaphisicae» tiene por objetivo examinar la noción 
de modo ofrecida por Francisco Suárez. En él se recurre a una dilucidación sobre el concepto de modo en relación al de ente y unidad. La separabilidad real y modal, junto con la potencia absoluta de Dios, son puntos clave para el objetivo de dicho ensayo. Un modo, a diferencia de un ente, es inseparable de aquel ente en virtud del cual tiene ser y unidad (ver p. 36).

«Cómo convertirse en otra persona sin dejar de ser uno mismo» es el nombre del tercer ensayo que representa el final del primer momento biográfico del libro de Hurtado. Este tiene un papel reconciliador y por lo tanto importante para los dos ensayos anteriores ya que, gracias a una noción de cambio, de modos y de unidad, se aboga por una teoría modal sobre el cambio de las personas. Hurtado parte del supuesto de que las personas estamos en constante cambio, y si es posible decir algo sobre la identidad personal, no se debe de perder de vista este hecho. Para concebir este cambio en la identidad personal Hurtado recurre al uso metafórico de persona como «rostro» del ser humano. De aquí sostiene que «persona» no es lo mismo que «ser humano»; sin embargo, la persona que somos no es algo aparte del ser humano que somos. Esto se entiende bajo una teoría modal delimitada en el ensayo anterior. En efecto, la persona (o el rostro) puede cambiar y ser otra persona o dejar de ser cierta persona, pero la persona seguirá siendo el mismo ser humano. El rostro es un modo que como tal es inseparable del ente humano, pero éste puede someterse al cambio. Así, el tercer ensayo puede parecer inconexo para el lector descuidado, sin embargo resulta una reconciliación de lo visto en los dos anteriores y un paso necesario para el segundo momento biográfico dedicado a la «doxología». 
De la tesis general del tercer ensayo que sostiene que una persona puede cambiar sin dejar de ser el mismo ser humano, se sigue que muchos «cambian sus creencias más básicas, sus deseos más profundos e incluso sus proyectos de vida» (p. 43); algo similar al alcohólico en rehabilitación que decide cambiar su modo de enfrentarse al mundo, o el científico que cambia de paradigma como producto de una investigación.

El cuarto ensayo, al que se le adjudica el título del libro «Por qué no soy falibilista» corresponde a un intento por contraponer al sentido común con la disciplina que postula que cualquiera de nuestras creencias puede resultar falsa, para, al mismo tiempo, sostener que no hay buenas razones para sostener la postura mencionada. Quizá el paso más importante para esta tarea es caracterizar al falibilismo como una postura revisionista que, al llevar la carga de la prueba (ver p. 60), oscurece nuestra situación epistémica ya que planta una duda en cualquiera de nuestras creencias. Caso aparte el del creyente ordinario que parte de la premisa «algunas de nuestras creencias pueden resultar ser falsas» que evita la tarea revisionista de poner en duda cualquier creencia. Hurtado optará por la segunda postura mostrando las dificultades de los razonamientos a favor de la primera bajo dos clases de argumentos: los epistemológicos y los éticos. De la segunda clase se siguen aportaciones por demás interesantes al tan común y oscuro tema de la tolerancia; pues ser falibilista no implica ser tolerante ya que el creyente ordinario puede ser tolerante, sin ser necesariamente partidario del falibilismo. El argumento epistemológico se basa en que «se puede hablar del mundo sin tener teorías científicas sobre el mundo» (p. 55); por lo que podríamos afirmar que hay creencias ordinarias que corresponden al lenguaje común y 
que pueden ser no falibles al ser independientes (es decir, no participar en el holismo doxástico que proponía Quine) de las teorías científicas sobre el mundo. Tanto del argumento ético, como del epistemológico Hurtado desprende y muestra la debilidad dialéctica del falibilismo en relación al dogmatismo y al escepticismo; de este último, Hurtado dedicará un apéndice con el objetivo de diferenciarlo de la postura de que cualquiera de nuestras creencias puede resultar falsa.

¿Por qué tomar a este ensayo como carta de presentación de los demás? Posiblemente porque en un par de decenas de páginas se logran englobar los temas tratados en los anteriores y en los siguientes ensayos. «Por qué no soy falibilista» es el puente entre un momento biográfico y otro. El puente que va desde un estudio ontológico sobre el cambio, el modo y la identidad personal, a una «doxología» que presupone la identidad personal como algo inseparable a la condición epistémica.

«Dudas y sospechas» es el nombre del quinto y penúltimo ensayo. En él, después de haber aceptado considerar algunas creencias como falsas, Hurtado expone por medio de filosofía del lenguaje ordinario una crítica a la epistemología tradicional al postular que una duda no es siempre algo opuesto e incompatible a una creencia. En efecto, se puede creer en algo y aún así tener algunas dudas al respecto. A esta clase de dudas Hurtado las denomina «dudas-A» y son distintas a las dudas plenas donde la creencia de $p$ es negada por la implantación de una duda. El ensayo está conformado, para estas distinciones, por grados de creencia que permiten posteriormente discernir sobre los conceptos de «duda», «creencia», «certeza» $\mathrm{y}$ «sospecha» en el bien logrado ejemplo del celoso Otelo; personaje que pasa de una «sospecha» 
a una «duda-A» gracias al testimonio de Yago respecto a la fidelidad de Desdémona.

El último ensayo denominado «Notas sobre De Utilitate Credendi» pretende mostrar el aporte de Agustín a la teoría general de la creencia precisamente en cuanto al sentido de creer sin ver (i.e. sin saber) y en cuanto a la posibilidad de una duda. Para este fin es necesaria la mención de las creencias por tradición, testimonio e institución que Agustín utiliza de manera apologética para la religión Católica en contra de los maniqueos que postulaban que para creer algo, hay que verlo. Agustín, a su vez, acude a una reducción al absurdo práctico (ver p. 79), para mostrar que es imposible dudar de lo que nos acontece en la vida diaria, y de lo que nos es común creer. Por ejemplo, dice Agustín, sólo los insensatos pueden dudar que su madre sea su madre real ya que «no debemos dudar sin causa ni razón» (p. 80). Caso paradigmático resulta el del amigo del que podemos dudar bajo ciertas condiciones si en efecto es tal. El punto a resaltar sería un intento de Hurtado por clarificar que existen creencias ordinarias que no se cuestionan a menos que se tenga alguna razón para implantar una duda. Lo anterior resulta agradable para el libro en general pues atiende al preocupante asunto de los celos, las sospechas y las dudas mencionadas en el anterior ensayo. Otelo dudó hasta que tuvo una razón para hacerlo; en este caso, el «testimonio» de Yago. Además, el ensayo en cuestión resulta, bajo la lectura contemporánea y laica de Hurtado, una forma satisfactoria de concluir el libro. Ya que, al haber pasado por un estudio sobre el cambio, los modos, la identidad personal, el cambio en nuestras creencias, las dudas, sospechas y certezas, nos damos cuenta de la practicidad con la que el estudio de los estados epistémicos 
ha sido abordado en los ensayos y su acomodo obsequiados por Guillermo Hurtado.

Cierto es que Por qué no soy falibilista y otros ensayos filosóficos de Guillermo Hurtado no manifiesta, por lo menos de manera explícita, un eje temático entre los ensayos, sin embargo, gracias al trabajo en conjunto de un buen cuidado editorial propio de Los Libros de Homero y la perspicacia del autor, el texto que nos obsequian resulta una merecida obra biográfica de claro interés filosófico.

Luis Ángel García Muñoz

Maestría en Filosofía Universidad Nacional Autónoma de México 
